\title{
PROGRAMA DE TRIAGEM AUDITIVA SELETIVA \\ EM CRIANÇAS DE RISCO EM UM SERVIÇO DE SAÚDE AUDITIVA DE SÃO PAULO
}

\author{
Selective hearing screening program for children \\ under risk at a hearing health service in São Paulo
}

\author{
Monica de Almeida Cardillo-Martins Scaziotta ${ }^{(1)}$, \\ Isabela Freixo Côrtes de Andrade(2), Dóris Ruthi Lewis(3)
}

\begin{abstract}
RESUMO
Objetivo: descrever a população de neonatos encaminhada para um programa de triagem auditiva seletiva, caracterizando e comparando o grupo de lactentes que compareceram à triagem (grupo I) com o grupo de lactentes que não compareceu (grupo II). Método: a amostra foi constituída por 55 lactentes, provenientes de uma maternidade de São Paulo. A metodologia incluiu a análise de prontuários e entrevistas com as mães. Foram variáveis do estudo: idade da alta hospitalar, resultado da triagem auditiva, resultado do diagnóstico, o tempo entre a alta hospitalar e a triagem, o tempo entre a alta hospitalar e o diagnóstico e, por fim, o tempo entre triagem e diagnóstico. Além destes aspectos, as características socioeconômicas e culturais dos grupos e os indicadores de risco foram analisados. Resultados: foram encaminhados 55 lactentes e o comparecimento à triagem auditiva foi de $76 \%$ (42). A média de idade da alta hospitalar foi de 38 dias, da triagem auditiva foi de 42 dias e do diagnóstico foi de 95,1 dias. A média do tempo entre a alta e a triagem foi de 13 dias e da alta e diagnóstico de 40,8 dias. O grupo que compareceu à triagem apresentou peso menor, maior período de internação na UTI, maior número de indicadores de risco, maior renda familiar por pessoa e maior número de consultas pré-natal em comparação aos que não compareceram. Conclusões: as crianças que mostraram maior adesão à realização da triagem auditiva neonatal seletiva foram aquelas cujas mães compareceram a um maior número de consultas no pré-natal, as que apresentaram maior ocorrência de indicadores de risco, maior tempo de internação e quando as informações na maternidade mostraram-se mais efetivas.
\end{abstract}

DESCRITORES: Audição; Perda Auditiva; Triagem Neonatal; Recém-nascido

\section{INTRODUÇÃO}

No decorrer dos anos, de forma gradativa e progressiva, no Brasil, a Política Nacional de Atenção à Saúde Auditiva vem sendo implantada em diversos estados. Políticas públicas de

(1) Fonoaudióloga; Mestre do Programa de Estudos Pós-Graduados em Fonoaudiologia da PUC-SP.

(2) Fonoaudióloga; Mestre e Doutoranda do Programa de Estudos Pós-Graduados em Fonoaudiologia da PUC-SP.

(3) Fonoaudióloga; Professora Titular do Programa de Pós Graduação em Fonoaudiologia e Faculdade de Fonoaudiologia da PUC-SP; Doutora em Saúde Pública pela Universidade de São Paulo.

Conflito de interesses: inexistente saúde devem buscar alternativas e soluções para a saúde auditiva neonatal da população, visando, assim, obter um equilíbrio em relação ao orçamento público a fim de promover o acesso da população a estes serviços ${ }^{1}$.

Em países desenvolvidos, desde 1993, iniciouse o processo de implantação de triagem auditiva neonatal universal (TANU), sendo que, em alguns desses, esta prática já se encontra plenamente desenvolvida. No entanto, na maioria dos países em desenvolvimento, como exemplo o Brasil, não foi possível iniciar esta ação mais ampla da TANU devido às dificuldades no financiamento de tais programas ${ }^{2}$. 
Em 28 de setembro de 2004, no Brasil, o Ministério da Saúde criou a Política Nacional de Atenção à Saúde Auditiva, conforme Portaria GM/ MS n²073. Dentre as ações de Saúde Auditiva na Atenção Básica, destaca-se o atendimento integral à população, com ações de promoção da saúde, prevenção e identificação precoce de problemas auditivos. Os atendimentos de média e de alta complexidade deveriam incluir as triagens auditivas e o monitoramento da audição, além do diagnóstico, tratamento clínico e reabilitação com fornecimento de aparelho de amplificação sonoro individual (AASI), e terapia fonoaudiológica ${ }^{3}$.

Maternidades que não possuam o serviço de TANU poderiam encaminhar os lactentes para os Serviços de Média Complexidade. Desta forma, as crianças se beneficiariam ao realizar a triagem auditiva fora da maternidade, em um Centro de Referência, e, se necessário, realizar o diagnóstico e a intervenção fonoaudiológica ${ }^{4}$. Apesar da facilidade de se realizar a TANU antes da alta hospitalar, nos casos onde essa ação não é possível, os lactentes de risco poderiam ser encaminhados após a alta hospitalar para serviços que dispõem de equipamento para realização deste procedimento, pois estes têm maior ocorrência de perdas auditivas de qualquer tipo (condutiva, sensórioneural e espectro da neuropatia auditiva). Essa triagem neste artigo será chamada de triagem auditiva seletiva e externa (TASE), pois foi realizada somente em neonatos de risco no centro de referência.

Para que os neonatos encaminhados para realização da triagem auditiva compareçam em Centros de Referência é preciso que os profissionais envolvidos neste processo conheçam as peculiaridades da população a ser avaliada. Sabe-se que as condições de gestação e nascimento da criança (que vão determinar baixo ou alto risco para deficiência auditiva $)^{5}$ e a escolha dos procedimentos ${ }^{6}$ estão entre os principais fatores que podem interferir nos resultados dos programas de triagem auditiva. No entanto, fatores como a idade, local de realização dos testes ${ }^{7}$ e características socioeconômicas e culturais $^{8}$ da população, também, podem interferir nos achados.

Os comitês recomendam que a triagem auditiva seja realizada até os 3 meses e o diagnóstico com início da intervenção até os 6 meses de idade ${ }^{9-12}$. No entanto, crianças com indicadores de risco para deficiência auditiva (IRDA) tendem a ser triadas e diagnosticadas mais tardiamente do que as de baixo risco, pois há necessidade de aguardar a estabilidade do quadro vital para o inicio das avaliações ${ }^{12}$.

Segundo o Joint Committee on Infant Hearing( $1994)^{10}$, aproximadamente $50 \%$ dos recémnascidos que não que não obtém resultado "passa" na primeira triagem, deixam de ter um acompanhamento apropriado para confirmar a perda auditiva, e consequentemente, iniciar a intervenção precoce.

A partir dessa perspectiva, este estudo tem por objetivo descrever a população de neonatos com IRDA encaminhada para um programa de triagem auditiva seletiva externa, em um serviço de saúde auditiva de São Paulo, caracterizando e comparando o grupo de lactentes que compareceram à triagem, com outro que não aderiu ao programa proposto pela maternidade.

Os objetivos específicos são comparar os grupos, com relação às seguintes variáveis:

- Idade da triagem auditiva, tempo entre a alta hospitalar e triagem, tempo entre a alta hospitalar e o diagnóstico, tempo entre triagem e diagnóstico e idade do diagnóstico;

- Indicadores de risco; e

- Características socioeconômicas e culturais.

\section{MÉTODO}

Este estudo foi prospectivo, realizado em um centro de referência de saúde auditiva de São Paulo credenciado ao SUS como média e alta complexidade.

Os lactentes foram encaminhados de uma maternidade considerada referência no atendimento a gestante de risco da cidade de São Paulo, situada na região leste da cidade. A maternidade tem uma média de 200 nascimentos/mês; estimase que $20 \%$ da população atendida seja gestação de risco; e cerca de 4 a $5 \%$ sejam neonatos de risco para deficiência auditiva, com grande ocorrência de prematuridade e sífilis congênita.

Foram incluídos neste estudo os neonatos nascidos entre fevereiro e agosto de 2006. A população foi selecionada pelas equipes médica e de enfermagem do hospital, seguindo os critérios de risco para deficiência auditiva segundo o Joint Committee on Infant Hearing ${ }^{10,11}$.

A amostra foi constituída por 55 lactentes, e distribuida em dois grupos: I - crianças que compareceram à triagem, $42(76 \%)$ e; II- crianças que não compareceram, 13 (24\%).

Para a caracterização do grupo que compareceu à triagem os dados foram coletados por meio de entrevista com a mãe (ou responsável) e análise do cartão de alta da maternidade. Para caracterização do grupo II, que não compareceu à triagem foram coletados dados por meio de entrevista telefônica com as mães, análise do prontuário do hospital e análise da Declaração de Nascidos Vivos.

Os dados coletados para análise foram: presença de IRDA, idade da alta hospitalar, região de moradia, inclusão no mercado de trabalho, 
acesso ao serviço de saúde, renda familiar, grau de escolaridade da mãe, número de filhos e número de consultas pré-natal. Ao grupo que compareceu à triagem auditiva, foram analisados também: idade da triagem auditiva, tempo entre a alta hospitalar e triagem, tempo entre a alta hospitalar e o diagnóstico, tempo entre triagem e diagnóstico e idade do diagnóstico.

$\mathrm{Na}$ triagem auditiva, foi utilizado o analisador automático de emissão otoacústica por estímulo transiente, produto de distorção e potencial evocado auditivo de tronco encefálico, modelo AccuScreen Pro TDA, marca GN Resound.

No caso do lactente apresentar resultado "passa" em todos os procedimentos realizados, houve orientação e encaminhamento para o monitoramento anual do desenvolvimento auditivo, sendo inseridos no Programa de Acompanhamento de Crianças de Risco do CeAC/DERDIC/PUCSP; no caso de "falha" em um ou mais procedimentos, houve encaminhamento para o reteste em 15 dias. No caso de "falha" em pelo menos um procedimento do reteste, houve encaminhamento para o diagnóstico, com avaliação otorrinolaringológica, realização de emissão otoacústica evocada por estímulo transiente, produto de distorção, potencial evocado auditivo de tronco encefálico - pesquisa de nível mínimo de resposta eletrofisiológica com estímulo clique, por via aérea e via óssea, e encaminhamentos necessários.

O protocolo ao qual os lactentes foram submetidos foi enviado para apreciação e aprovação pelo Comitê de Ética da Pontifícia Universidade Católica de São Paulo, sob o número de aprovação 0007/2005. As mães que compareceram à triagem auditiva foram informadas sobre a importância da pesquisa por meio do termo de consentimento livre e esclarecido e assinaram uma autorização, dessa forma, confirmando a autorização da participação neste estudo.

$\mathrm{Na}$ analise estatística, foram calculadas as medidas descritivas (média, desvio padrão, mínimo, mediana e máximo) dos dois grupos da amostra. Construíram-se tabelas contendo os valores estatísticos das variáveis observadas nos dois grupos. O critério de significância adotado para as variáveis estudadas foi $p<0,05\left(^{*}\right)$.

\section{RESULTADOS}

O grupo I foi formado por 42 (76\%) lactentes, sendo 17 (40\%) do sexo feminino e 25 (60\%) do sexo masculino; o grupo II foi formado por $13(24 \%)$ lactentes, sendo $7(53,9 \%)$ do sexo feminino e 6 $(46,1 \%)$ do sexo masculino.
A Figura 1 mostra os resultados dos 42 lactentes triados, $32(76,2 \%)$ passaram em todos os testes e foram encaminhados para o monitoramento anual do desenvolvimento auditivo; 10 (23,8\%) lactentes apresentaram falha em pelo menos um dos procedimentos de triagem , sendo que $8(19,0 \%)$ foram encaminhados para o reteste e $2(4,8 \%)$ foram encaminhados direto para o diagnóstico. Destes encaminhados para diagnóstico, 1 (2,4\%) apresentava malformação de pavilhão auricular unilateral e $1(2,4 \%)$ falhou na pesquisa do RCP.

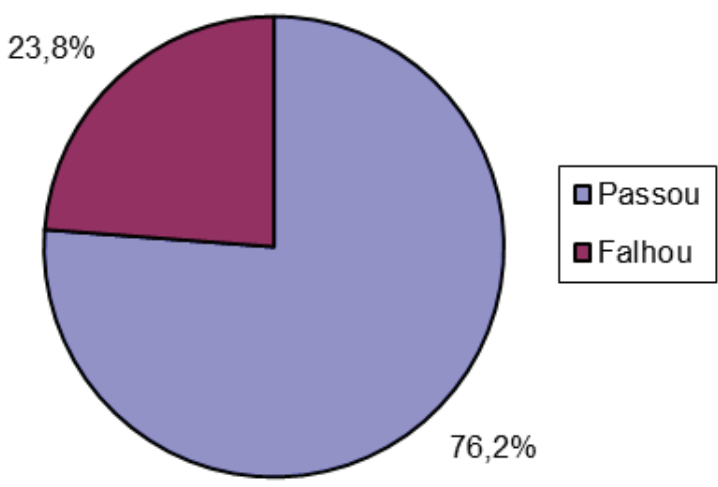

Figura 1 - Resultados da triagem auditiva (teste), segundo os critérios de passa/falha estabelecidos

Das 8 crianças encaminhadas para o reteste, 1 $(12,5 \%)$ não compareceu. Foi realizado o contato telefônico com a mãe dessa criança , remarcando a avaliação por três vezes, porém, a criança novamente não compareceu. Das 7 crianças que retornaram para o reteste, $1(12,5 \%)$ havia falhado na pesquisa de EOAT bilateralmente e passou no reteste; $6(87,5 \%)$ continuaram apresentando falha em pelo menos um procedimento e foram encaminhadas para o diagnóstico.

Dos 8 lactentes encaminhados para o diagnóstico, $2(25 \%)$ apresentaram resultados normais e 6 (75\%) apresentaram perda auditiva do tipo condutiva. Após o diagnóstico, observou-se que a prevalência de perda auditiva na população estudada foi de $14,3 \%$ (aproximadamente 1:7 nascimentos). Nessa amostra, não foi encontrado nenhum lactente com perda auditiva do tipo sensorial.

$\mathrm{Na}$ Tabela 1, descrevem-se as medidas estatísticas para idade da alta (dias), idade da triagem (dias), idade do diagnóstico, diferença de tempo entre a alta hospitalar e a triagem (dias), diferença de tempo entre a alta e o diagnóstico e diferença de tempo entre a triagem e o diagnóstico.

A média de idade da alta hospitalar foi de $38( \pm$ $22,3)$ dias e da triagem auditiva foi de $42( \pm 23,9)$ 
dias. A média de tempo entre a alta e a triagem foi de $13( \pm 9,3)$ dias. O lactente que demorou menos tempo realizou a triagem auditiva em um dia depois de ter recebido alta. $\mathrm{O}$ lactente que demorou mais tempo realizou após 35 dias.
A média de tempo entre a alta e o diagnóstico foi $40,8( \pm 20,9)$ dias. A média de idade dos lactentes que realizaram o diagnóstico foi 95,1 dias ou aproximadamente 3 meses e $5( \pm 44,6)$ dias.

Tabela 1 - Estatísticas para idade da alta (dias), idade da triagem, diferenças de tempos entre a alta hospitalar e a triagem auditiva (dias), idade do diagnóstico, variáveis entre tempo da alta e do diagnóstico; e tempo da triagem e diagnóstico

\begin{tabular}{lcccccc}
\hline Dias & N & Média & DP & Mediana & Mínimo & Máximo \\
\hline Idade da Alta & 38 & 35,2 & 22,3 & 31 & 2 & 97 \\
Idade da Triagem & 42 & 50,0 & 23,9 & 49 & 16 & 123 \\
Média de Tempo entre Alta e Triagem & 38 & 13,0 & 9,3 & 10 & 1 & 35 \\
Idade do Diagnóstico & 8 & 95,1 & 44,6 & 115,0 & 31 & 149 \\
Tempo entre Alta e Diagnóstico & 8 & 40,8 & 20,9 & 37,5 & 12 & 76 \\
Tempo entre Triagem e Diagnóstico & 8 & 35,5 & 30,6 & 24,5 & 6 & 86 \\
\hline
\end{tabular}

Legenda: - Teste estatístico: Medidas descritivas média, desvio padrão, mediana. mínimo e máximo.

- Número (N); Desvio Padrão (DV).

As Tabelas 2 e 3 ilustram as características dos grupos I e II quanto ao tempo de internação em UTI (dias), peso ao nascimento (gramas) e número de IRDA.

Das 42 crianças do grupo I, $37(88,1 \%)$ necessitaram de internação na UTI e 5 (11,9\%), vieram encaminhadas do alojamento conjunto. O tempo médio que os lactentes ficaram internados foi de $25,6$ ( $\pm 19,1)$ dias. Para o grupo II, a média de tempo de internação na UTI foi de 5,1 $( \pm 3,9)$ dias.
A comparação entre os dois grupos mostrou que o grupo I apresentou tempo de internação significantemente maior que o grupo II $(p=0,01)$.

A média de peso apresentada pelos lactentes que compareceram à triagem foi de $1840 \mathrm{~g}( \pm 793)$; e para o grupo que não compareceu foi de $2270 \mathrm{~g}$ $( \pm 535)$. Na comparação entre os grupos, observouse que os lactentes que realizaram a triagem apresentaram peso significantemente menor que os lactentes que não a realizaram $(p=0,014)$.

Tabela 2 - Estatísticas para tempo de UTI (dias) e para a variável peso (gramas), para os dois grupos segundo o comparecimento à triagem auditiva

\begin{tabular}{cccccccc}
\hline Grupo & N & Média & DP & Mínimo & Mediana & Máximo & $\mathbf{p}$ \\
\hline I - UTI & 37 & 25,6 & 19,1 & 1 & 20 & 81 & $\mathbf{p}<\mathbf{0 , 0 0 1}$ \\
II - UTI & 13 & 5,1 & 3,9 & 1 & 4 & 16 & \\
I - PESO & 42 & 1840 & 793 & 705 & 1635 & 3610 & $\boldsymbol{p}=\mathbf{0 , 0 1 4}$ \\
II -PESO & 13 & 2270 & 535 & 1810 & 2070 & 3730 & \\
\hline
\end{tabular}

Legenda: - Teste estatístico: Kruskal-Wallis - Medidas descritivas da média, desvio padrão, mediana, mínimo e máximo.

- Critério de significância = valor de $p<0,05$ (UTI: $p=0,001$ - Grupo I apresentou tempo de internação significantemente maior que o grupo II e Peso: $p=0,014$ - Grupo I apresentou peso significantemente menor que o grupo II);

- Número (N); Desvio Padrão (DV).

O grupo que compareceu para a realização da triagem auditiva apresentou número de indicadores de risco significantemente maior que o grupo que não compareceu $(p=0,03)$. A tabela 3 mostra o número e a frequência de indicadores de risco para os dois grupos segundo o comparecimento à triagem. O grupo I apresentou, em média, dois indicadores de risco; enquanto o grupo II apresentou 1,4 indicadores. Nota-se que $100 \%$ dos neonatos que não compareceram à triagem apresentaram um ou dois indicadores de risco; por sua vez, 31\% (13) dos neonatos que realizaram a triagem apresentaram de três a quatro indicadores de risco. 
Tabela 3 - Estatísticas descritivas para número de indicadores de risco nos dois grupos definidos segundo o comparecimento à triagem

\begin{tabular}{ccccccc}
\hline Triagem & N & Média & DP & Mínimo & Mediana & Máximo \\
\hline I & 42 & 2,0 & 0,9 & 1 & 2 & 4 \\
II & 13 & 1,4 & 0,5 & 1 & 1 & 2 \\
\hline
\end{tabular}

Legenda: - Teste estatístico: Kruskal-Wallis - Medidas descritivas da média, desvio padrão, mediana, mínimo e máximo.

- Critério de significância $=$ valor de $p<0,05$ (Comparecimento à triagem: $p=0,03$ - Grupo I apresentou comparecimento à triagem significantemente maior que o grupo II)

- Número (N); Desvio Padrão (DV).

Na Tabela 4, é possível visualizar a comparação entre a renda familiar, o número de filhos e a renda familiar por pessoa.

Dos 42 responsáveis que compareceram à triagem, 5 (17\%) não souberam informar a renda. A renda familiar média foi de $\mathrm{R} \$ 868,50$. Não houve diferença significante entre as rendas familiares médias $(p=0,056)$. As mães que compareceram à triagem tinham, em média, 1,8 filhos $( \pm 0,8)$; enquanto o grupo que não compareceu tinha em média 3 filhos $( \pm 2,0)$. As mães do grupo I apresentaram número de filhos significantemente menor que as mães do grupo II $(p=0,017)$.

A renda familiar média por pessoa do grupo I foi significantemente maior $(p=0,03)$ que o grupo II. Enquanto o grupo I apresentou renda familiar por pessoa de $\mathrm{R} \$ 235,00( \pm 174,5)$; o grupo II apresentou de $\mathrm{R} \$ 131,60( \pm 64,0)$.

Tabela 4 - Estatísticas descritivas para renda familiar e renda familiar/pessoa (reais) para os dois grupos segundo o comparecimento à triagem

\begin{tabular}{cccccccc}
\hline Variável & Grupo & N & Média & DP & Mediana & Mínimo & Máximo \\
\hline \multirow{2}{*}{ Renda } & II & 11 & 532,7 & 178,2 & 280 & 500 & 850 \\
& I & 37 & 868,5 & 653,9 & 650 & 300 & 3200 \\
\hline \multirow{2}{*}{ Renda/pessoa } & II & 11 & 131,6 & 64,0 & 70 & 124 & 283,3 \\
& I & 37 & 235,0 & 174,5 & 176,7 & 75 & 833,3 \\
\hline
\end{tabular}

${ }^{*} p=0,056$

Legenda: - Teste estatístico: Kruskal-Wallis - Medidas descritivas da média, desvio padrão, mediana, mínimo e máximo.

- Critério de significância $=$ valor de $p<0,05$ (Renda Familiar: $p=0,056$ - Grupo I apresentou renda familiar média por pessoa maior que o grupo II).

- Número (N); Desvio Padrão (DV).

Sobre a inclusão no mercado de trabalho, observou-se o acesso aos serviços de saúde dos grupos I e II segundo o comparecimento à triagem auditiva. Das 42 mães do grupo I, 16,7\% referiram trabalhar (registrada ou autônoma); e 83,3\%, não trabalhar; no grupo II, 36,7\% referiram trabalhar; e $63,3 \%$, não trabalhar. O acesso ao serviço de saúde particular foi observado apenas no grupo I, sendo que $12 \%$ relataram possuir convênio. A totalidade das mães que não compareceu referiu ter acesso somente ao serviço público de saúde.

Observou-se a distribuição da porcentagem quanto ao número de consultas pré-natal e grau de escolaridade das mães dos grupos I e II. A maioria $(95,2 \%)$ das mães do grupo que compareceu à triagem auditiva referiu ter realizado mais de quatro consultas pré-natal; enquanto que $56,2 \%$ das mães que não compareceram realizaram o mesmo número de consultas. Quando comparadas, as mães do grupo I realizaram mais consultas que as mães do grupo II, sendo esta diferença estatisticamente significante $(p=0,013)$. Quando comparado o grau de escolaridade dos grupos, não foi observada diferença significante $(p=0,194)$.

$\mathrm{Na}$ Tabela 5, são apresentados os dados referentes ao profissional do hospital que fez o encaminhamento para a triagem auditiva segundo informações dos responsáveis pelas crianças dos dois grupos. Quanto ao grupo I, foram analisadas as respostas de 38 responsáveis, excluindo as quatro crianças que realizaram a triagem, ainda internadas. Do total, $22(57,9 \%)$ afirmaram que o encaminhamento foi feito pelo(a) médico(a), 14 $(36,8 \%)$ revelaram que o encaminhamento foi feito 
por um profissional da enfermagem e 2 (5,3\%) não souberam informar. Todos os responsáveis que souberam indicar o profissional que os encaminhou, souberam também referir o nome, desse modo, demonstrando vínculo com o mesmo. Para o grupo II, $6(54,5 \%)$ não souberam referir e 5 $(45,5 \%)$ disseram ter sido encaminhados pelo(a) médico(a).

Tabela 5 - Distribuição de frequências e porcentagens do profissional que fez o encaminhamento para a triagem auditiva, para os dois grupos

\begin{tabular}{ccccc}
\hline Profissional & Frequência I & $\%$ & Frequência II & $\%$ \\
\hline Médico(a) & 22 & 59,7 & 5 & 45,5 \\
Enfermeiro(a) & 14 & 36,8 & 0 & 0 \\
Não sabe & 2 & 5,3 & 6 & 54,5 \\
Total & 38 & 100 & 11 & 100 \\
\hline
\end{tabular}

\section{DISCUSSÃO}

Os achados do presente estudo sugerem que a triagem auditiva seletiva externa realizada em centros de referência é viável, visto que $76 \%$ das crianças encaminhadas compareceram ${ }^{13}$ e as idades da triagem e diagnóstico estão de acordo com o preconizado na literatura ${ }^{9-12}$. O encaminhamento para a realização da triagem auditiva neonatal fora da maternidade pode ser uma alternativa para a demanda proveniente de hospitais que não possuam o serviço. Observou-se que os lactentes que não realizaram a triagem eram os de menor risco auditivo (menos tempo de internação, peso maior e menor número de indicadores de risco) ${ }^{14}$.

Em países como o Brasil, que apresenta grande diversidade regional com cidades e hospitais de pequeno porte, nos quais, a TANU não está inserida, a implantação de programas de TASE poderia ser uma estratégia para maximizar a demanda? ${ }^{7}$.

Outro aspecto significante na pesquisa foi que os resultados corroboram com os achados de estudos que utilizaram como procedimentos de triagem a pesquisa de EOAPD e avaliação comportamental, e encaminharam $26,7 \%$ de lactentes de alto risco para o diagnóstico ${ }^{15}$. Porém, é importante citar que o índice de encaminhamento para diagnóstico nesta pesquisa $(26,7 \%)$ foi inferior ao obtido em outro estudo de avaliação auditiva em recémnascidos $(32 \%)^{14}$.

Do grupo de crianças que falhou na triagem auditiva após o teste e reteste, $75 \%$ apresentavam perda auditiva condutiva. Estes dados estão de acordo com os achados de pesquisadores que diagnosticaram $63,6 \%$ de perdas condutivas nas crianças que haviam falhado na triagem auditiva ${ }^{16}$.
O número de falsos positivos foi $25 \%$ (2 crianças). Estes achados discordam do estudo ${ }^{17}$ que diagnosticou perda auditiva em $60 \%$ das crianças encaminhadas para o diagnóstico, com índice de falso positivo de $40 \%$ e concordam com a pesquisa ${ }^{18}$ que diagnosticou perda em $80,98 \%$ das crianças encaminhadas para o diagnóstico com índice de falsos positivos de 19,02\%. Mesmo assim está bem elevado em relação aos 3\% ou 4\% recomendados pelo Comitê Brasileiro sobre Perdas Auditivas na Infância (CBPAI) ${ }^{11}$. As idades da triagem e do diagnóstico observadas neste estudo estão de acordo com o que recomendam os comitês: triagem até os 3 meses de idade (90 dias) e diagnóstico e intervenção até os 6 meses (180 dias) ${ }^{9-12}$. Alguns estudos observaram idade média da triagem menor ${ }^{19-21}$, porém estes programas de triagem foram realizados antes da alta hospitalar.

Pesquisadores em 2005 realizaram a triagem auditiva no primeiro retorno de consulta pós-alta hospitalar em lactentes de alto risco e obtiveram idade média maior que o encontrado neste estudo: 73,5 dias (aproximadamente 2 meses e 13 dias) ${ }^{(14)}$.

Observou-se, também, que o tempo de internação na unidade de terapia intensiva foi um fator que teve influência direta na idade da triagem e no diagnóstico. No grupo I, 88,1\% necessitaram de internação na UTI, em um tempo médio de 25,6 dias. A diferença de idade do diagnóstico entre as crianças de alto e baixo risco deve-se à necessidade de estabilizar o quadro vital das crianças de risco, antes de realizar a triagem ${ }^{12,21,22}$.

O diagnóstico audiológico foi realizado, em média, 40 dias após a alta hospitalar e, aproximadamente, 35 dias após a triagem auditiva. Há estudos que realizaram a triagem auditiva, no primeiro retorno de consulta pós-alta hospitalar, em lactentes de alto risco, e obtiveram idade 
média maior que o encontrado neste estudo, como 73,5 dias ${ }^{14}$.

Quanto ao número de consultas pré-natal, verificou-se que as mães do grupo I realizaram mais consultas que as mães do grupo II, sendo esta diferença estatisticamente significante. Esses resultados podem ser comparados com uma pesquisa realizada em Recife, nas maternidades públicas, cujo objetivo era descrever as características socioeconômicas das mães. Foi concluído que 36\% da amostra realizaram de cinco a oito pré-natais e $7 \%$ realizaram mais de onze. Conclui-se que grande parte das gestantes também realizara acompanhamento pré-natal de forma adequada. $O$ número de consultas pré-natais é um fator bastante importante e pode estar associado aos cuidados com a saúde da mãe e da criança ${ }^{23}$.

Os vínculos estabelecidos entre as equipes do centro de referência e do hospital e entre as famílias e a equipe do hospital mostraram-se importantes no comparecimento à triagem. De um lado, o hospital forneceu informações a respeito do recém-nascido e da família; de outro, o centro de referência manteve a equipe atualizada quanto ao encaminhamento, resultado e conduta da triagem auditiva.

A proximidade entre a equipe do hospital e os pais também se mostrou relevante no comparecimento à triagem auditiva. Os pais que compareceram mostraram conhecer e confiar na equipe do hospital e acatar o encaminhamento e as orientações. Um total de $94,7 \%$ dos pais do grupo I souberam informar que o encaminhamento foi feito pelo(a) médico(a) ou pela enfermagem, em contrapartida, no grupo II, apenas $45,5 \%$ indicaram 0 encaminhamento devido a algum profissional do hospital. Estes dados mostram o quanto a proximidade da equipe do hospital é importante para ajudar no encaminhamento desses neonatos para a realização da triagem auditiva seletiva.

A coleta de dados das condições socioeconômicas e culturais das famílias encaminhadas para a triagem pode auxiliar em novas estratégias para aumentar o comparecimento/adesão ao programa de identificação e intervenção precoce, e triagem auditiva ${ }^{8,24}$. Os resultados da amostra apontam que aprofundar o conhecimento das características da população a que se destina o programa de triagem pode facilitar o acesso e, assim, a divulgação e o conhecimento do serviço ${ }^{13,25,26}$.

De acordo com a presente pesquisa, sugere-se a realização de estudos com amostras maiores, e que aprofundem a discussão a respeito do comparecimento e retorno dos pacientes, pois o conhecimento das particularidades da população brasileira é de suma importância para a sensibilização e engajamento dos pais na triagem e diagnóstico das alterações auditivas.

\section{CONCLUSÃO}

Foi possível concluir que a triagem auditiva seletiva externa realizada em centro de referência se mostrou viável quando a TANU na maternidade ainda não estiver implantada.

As crianças que mostraram maior adesão à realização da TASE foram aquelas cujas mães compareceram a um maior número de consultas no pré-natal, as que apresentaram maior ocorrência de indicadores de risco, maior tempo de internação e quando as informações na maternidade mostraramse mais efetivas. Quanto maior o conhecimento e confiabilidade dos pais na equipe do hospital maior a chance de acatar o encaminhamento e as orientações em prol da saúde auditiva. 


\section{ABSTRACT}

Purpose: to describe the population of high risk newborns referred for a hearing screening program at a selective hearing health service, as well, to characterize and compare the group of infants who attended the screening (group I) and the other of infants who did not attend the screening (group II). Method: we performed a prospective study in a child hearing center. The sample consisted of 55 infants under high risk at a maternity hospital in Sao Paulo. The methodology included the examination of medical records and interviews with the mothers. We studied the age at the hospital, hearing screening, diagnosis, time between hospital discharge and screening, the time between hospital discharge and diagnosis and, finally, the time between screening and diagnosis. We compared the socioeconomic and cultural groups and the risk factors. Results: 55 infants were referred to the attendance and hearing screening was $76 \%$ (42). The mean age of hospital discharge was 38 days, the hearing screening was 42 days and the diagnosis was 95.1 days. The mean time between the discharge and screening was 13 days, between the discharge and the diagnosis was 40.8 days. The group attending the screening had lower weight, longer ICU stay, and greater number of risk factors, higher family income per person and more prenatal care than those who did not attend it. Conclusions: the children who showed greater adherence to the implementation of selective newborn hearing screening were those whose mothers attended a greater number of pre-natal cares, those that had increased incidence of risk factors, longer hospitalization and when the information in the maternity ward were more effective.

KEYWORDS: Hearing; Hearing Loss; Neonatal Screening; Infant, Newborn

\section{REFERÊNCIAS}

1. Côrtes-Andrade IF, Lewis DR. Distúrbio da Comunicação. 2008; 20(2): 279-81.

2. Olusanya, B.O.; Wirz, B.L.; Luxon, L.M. Hospitalbased universal newborn hearing screening for early detection of permanent congenital hearing loss in Lagos, Nigeria. International Journal of Pediatric Otorhinolaryngology. 2008; 72: 991-1001.

3. Política Nacional de Atenção à Saúde Auditiva. [cited 2006 sep 21]. Avaliable from: http://www. saude.gov.br

4. Pereira, PKS; Martins, AS; Vieira, MR; Azevedo, MF. Programa de triagem auditiva neonatal: associação entre perda auditiva e fatores de risco. Pró-Fono R Atual. 2007; 19(3): 237-40.

5. Garcia CFD, Isaac ML, Oliveira JAA. Emissão otoacústica evocada transitória: instrumento para detecção precoce de alterações auditivas em recém-nascidos a termo e pré-termo. Rev Bras Otorrinolaringol. 2002; 419 (68):334-52.

6. Mukari, SZ.; Tan, KY.; Abdullah, A. A pilot project on hospital-based universal newborn hearing screening: Lessons learned. Int. J. Pediatr. Otorhinolaryngol. 2006; 70: 843-51.

7. Swanepoel de W, Hugo R, Louw B. Infant hearing screening at immunization clinics in South Africa. Int J Pediatr Otorhinolaryngol. 2006; 70 (7): 1241-9.
8. Todd W. Universal newborn hearing screening: follow-up in two Georgia populations: newborn, mother and system correlates. Int $\mathrm{J}$ Pediatr Otorhinolaryngol. 2006; 70 (5): 807-15.

9. National Institutes of Health $(\mathrm{NIH})$. Early identification of hearing loss in infants and young children: consensus development conference on early identification of hearing loss in infants and young children. Consensus Steatment. 1993; 11 (1): 1-24.

10. Joint Committee on Infant Hearing. Position statement. Audiol Today. 1994; 6 (6): 6-9.

11. Comitê Brasileiro Sobre Perdas Auditivas $\mathrm{Na}$ Infância (CBPAI) - período neonatal. J Pediatria. 2001; 77:1.

12. Comitê Multiprofissional em Saúde Auditiva COMUSA. São Paulo, BR, 2009 [acesso em set 2009]. Disponível em: http://www.audiologiabrasil. org.br/pdf/COMUSA_final_17_maio2009.pdf

13. Prieve B, Dazell L, Berg A, Bradley M, Cacace A, Campbell D, DeCristofaro J, Gravel J, Greeberg $E$ et al. The New York State universal newborn hearing screening demonstration Project: outpatient outcome measures. Ear Hear. 2000; 21 (2): 104-17. 14. Sassada MMY, Ceccon MEJ, Navarro JM, Vaz FAC. Avaliação auditiva em recém-nascidos gravemente enfermos através do método de emissões otoacústicas evocadas transientes (EOAT) e audiometria de tronco cerebral (BERA). Pediatria. 2005; 27 (3): 154-62. 
15. Ruggieri-Marone M, Lichtig I, Marone S A M, Recém-nascidos gerados por mães com alto risco gestacional: estudo das emissões otoacústicas produto de distorção e do comportamento auditivo. Rev Bras Otorrinolaringol. 2002; 68 (2): 230-7.

16. Boone R T, Bower C M, Martins P F. Failed newborn hearing screens as presentation for otitis media with effusion in newborn population. Int $\mathrm{J}$ Pediatr Otorhinolaryngol. 2005; 69 (3): 393-7.

17. Helge T, Werle E, Barnick M, Wegner C, Ruhe $B$, Aust $G$, Rossi R. Two-tier screening process (TEOAE/AABR) reduces recall rates in newborn hearing screening. 2005. [citado em nov 2006]. Disponível em http://www.pubmed.com

18. Hood L J. Clinical applications of the auditory brainstem response. San Diego: Singular Publishing; 1998.

19. Ferreira R, Basile L, Munyo A, Añazo G. Emisiones otoacústicas em recién nacido com factores de riesgo auditivo. Arch Pediatr Urug. 2003; 74 (3): 11

20. Costa JMD, Almeida VF, Oliveira CACP, Sampaio ALL. Emissões Otoacústicas Evocadas por Estímulo Transiente e por Produto de Distorção em Recém-Nascidos Prematuros. Arq. Int. Otorrinolaringol. 2009;13(3):309-16.
21. Mattos Wilian Maduell de, Cardoso Luciana Ferreira, Bissani Clarice, Pinheiro Maria Madalena C, Viveiros Carla Mherlyn, Carreirão Filho Waldir. Análise da implantação de programa de triagem auditiva neonatal em um hospital universitário. Rev. Bras. Otorrinolaringol. 2009; 75(2): 237-44.

22. Pádua FGM, Marone S, Bento RF, Carvallo RMM, Durante AS, Soares JC, et al. Triagem Auditiva Neonatal: Um Desafio para sua Implantação. Arq. Int. Otorrinolaringol. 2005;9(3):189-94.

23. Simão R, Conto J, Fujinaga, Cl. Concepções de gestantes sobre a perda auditiva. Revista SalusGuarapuava-PR. 2008; 2(1): 23-35.

24. Masson GA, Françoso MFC. A prática do serviço social em um programa de triagem auditiva neonatal. Serviço Social \& Realidade, Franca. 2008;17(1): 322-39.

25. Griz SMS, Barbosa CP, Silva ARA, Ribeiro MA, Menezes DC. Aspectos demográficos e socioeconômicos de mães atendidas em um programa de triagem auditiva neonatal. Rev. soc. bras. fonoaudiol. 2010,15(2):179-83.

26. Lima GML, Marba STM, Santos, MFC. Triagem auditiva em recém nascidos internados em UTI neonatal. J Pediatr. 2006; 82 (2): 1-9.
http://dx.doi.org/10.1590/S1516-18462011005000049

RECEBIDO EM: 08/10/2010

ACEITO EM: 16/12/2010

Endereço para correspondência:

Isabela Freixo Côrtes de Andrade

Avenida Engenheiro Martins Romeo, 103/ 802

Ingá - Niterói -RJ

CEP: 24210-400

E-mail: isabelafono@yahoo.com.br 